Article

\title{
Double-Dielectric Microstrip Ultrahigh-Frequency Antenna for Digital Terrestrial Television
}

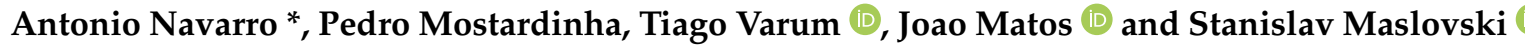 \\ Instituto de Telecomunicações and the Department of Electronics, Telecommunications and Informatics, \\ University of Aveiro, P-3810-193 Aveiro, Portugal; pedromostardinha@av.it.pt (P.M.); tiago.varum@ua.pt (T.V.); \\ matos@ua.pt (J.M.); stas@av.it.pt (S.M.) \\ * Correspondence: navarro@av.it.pt; Tel.:+351-234-377900
}

Received: 7 November 2020; Accepted: 26 November 2020; Published: 3 December 2020

Featured Application: Although Yagi-Uda antenna is more than 80 years old and is still globally the most used antenna for TV reception, we believe that there is still room for improvement. This article discusses a new antenna design for application in terrestrial TV broadcasting. With regard to the antenna's performance, our results are not limited to simulations. We present a prototype of a practical antenna supported by measurement results. This paper contributes to motivating and increasing research in the domain of antennas for digital terrestrial TV bands.

\begin{abstract}
In many countries, terrestrial broadcasting is the main delivery medium for television. In this paper, we propose a small-volume practical receiving antenna. Our design consists of a linear array of three vertically placed patch antennas which increase antenna gain. The antenna has a double-dielectric substrate (FR4 + air) in order to increase efficiency and bandwidth. In this paper, we also discuss simulations and practical results, and demonstrate that the proposed double-dielectric microstrip antenna is a viable design choice for digital terrestrial TV (DTT) reception. The designed antenna reached a gain of $10.5 \mathrm{dBi}$ at the desired central frequency of $754 \mathrm{MHz}$.
\end{abstract}

Keywords: TV broadcasting; terrestrial television; microstrip antenna; UHF

\section{Introduction}

In the modern world, switching from analog to digital television offers the user several advantages, such as high-definition (HD) video, six-channel surround audio (5.1), and data-broadcasting services. To make full use of the new audiovisual experience, the reception antenna must fulfill some requirements. Therefore, the design is very challenging for printed channel-bandwidth receiving antennas operating in the ultrahigh frequency band (UHF) [1].

Another well-known and important aspect of this challenge is that wireless communications suffer from interference that affects performance in terms of network capacity. This interference may arise, for example, from frequencies assigned to mobile networks (GSM, 3G, LTE, etc.) amplified by the input low-noise amplifier following the antenna. The upper limit of the digital television (DTV) band is next to either the GSM or LTE band [2]. Unlike other existing broadband antenna solutions, our novel proposed antenna avoids that interference because it has bandwidth close to a UHF IV and $\mathrm{V}$ band TV channel.

Printed antennas have several advantages, such as ease of manufacturing, reduced cost, and low volume. They are also thin in one dimension, making them suitable to operate in indoor enviroments and placed on the wall like a photograph or art frame. This makes such antennas a promising choice for DTV reception. However, a better solution in this application is to design an antenna with adjacent-channel rejection. Thus, the goal is to have the necessary bandwidth centered on the reception 
frequency while rejecting adjacent signals. In this framework, we designed an antenna that is intended for digital-terrestrial-television (DTT) reception.

The next section presents the state of the art. In Section 3, the problem of bandwidth is addressed. In Section 4, the antenna design is discussed, and its structure and dimensions are presented. In Section 5, antenna performance is substantively discussed, making use of several simulation results and practical measurements. Lastly, Section 6 concludes the paper.

\section{Related Work}

The Yagi-Uda antenna, commonly referred to as the Yagi antenna, is the most used antenna for TV reception. It was invented in 1926, so it is time to replace it with a novel design because of discoveries in new materials and structures since then. We need to move to a new type of antenna, but the research is tremendously challenging since the Yagi antenna's performance is very good.

Few antenna designs for DTV applications are presented in the literature. The antenna presented in [3] combines a planar inverted-F antenna (PIFA) and a loop antenna to achieve wideband operation in the UHF band. However, the operational bandwidth of the proposed PIFA-loop antenna is 460-870 MHz. Another interesting wideband antenna structure is a low-profile sinuous slot antenna constructed on a Rogers RO3206 substrate [4], where the gain varied from 2.5 to $6 \mathrm{dBi}$ in the frequency range of $0.53-4.86 \mathrm{MHz}$. As discussed above, these antennas require filtering due to the nearby frequencies/channels of the LTE band presented in this range, which can cause interference to the desired existing allocated services. Another limitation is that this PIFA-loop antenna is targeted to be used only indoors. In [5], a monopole antenna is proposed for DTV applications with a wide operational bandwidth (451-912 MHz). Likewise, this design is not suitable for the main requirement, which is the reduction of interference from the LTE band. The monopole antenna [5] has a gain of $1.7 \mathrm{dBi}$, which is very low compared to that of other DTV antennas. In order to increase the gain, a solution was proposed in [6] that was based on a unidirectional array of bowtie antennas with an incision gap. The measured gain for this antenna array was 13.4-16.1 dBi. The design demonstrated good half-power beam width (HPBW) in the vertical plane of $15^{\circ}$ from 470 to $862 \mathrm{MHz}$. Besides these features, the antenna also has return loss (RL) greater than $10 \mathrm{~dB}$ over a frequency range of 450-1014 MHz. A good example of a microstrip channel tuned antenna for Integrated Services Digital Broadcasting-Terrestrial (ISDB-T) was proposed in [7], but the gain was very low, around $-2.16 \mathrm{dBi}$.

Another challenging problem is how to reach the appropriate bandwidth of a microstrip antenna. For example, as was shown in [8], a bandwidth increase of about $13 \%$ could be obtained by proximity coupling. However, this method has great complexity of design and construction. The simplest and most direct method is to increase the thickness of the substrate, that is, to use a thicker dielectric with a lower dielectric constant. This enables an increase in bandwidth and efficiency; however, the possibility of surface-wave formation may decrease antenna radiation performance [9].

Extending this principle in order to achieve broader bandwidth, we propose a double-dielectric array antenna that adds another dielectric layer between patch substrate and ground plane. The second dielectric should have low permittivity, as discussed in Section 3. Alternatively, low-permittivity composite materials and metamaterials with a magnetic response, could be used to improve the bandwidth of microstrip antennas [10].

In this paper, we propose a new antenna that is well-matched at the frequency of interest and has the appropriate bandwidth, thus rejecting adjacent frequencies/channels such as the ones from the LTE band. The antenna was designed with linear polarization. Regarding the radiation pattern of the antenna, the main lobe had to be narrow enough to distinguish signals coming from different directions. Thus, by placing such antennas in a horizontal-array configuration, we could point the array radiation pattern maximum in the direction of the most favorable DTV broadcast signal (in terms of received power and modulation error rate), and simultaneously point the radiation pattern nulls in the direction of interfering signals. In this paper, we open a new path for motivating the research community to develop new antennas for TV reception. This paper takes a significant step towards 
that goal by proposing a new antenna for digital TV reception. Our design makes use of a microstrip patch antenna as a radiating element with applications at relatively low frequencies (in the DTV-UHF band). The advantage of the proposed design is that it can easily be integrated into printed circuit boards (PCBs). It is also versatile in adjusting the antenna resonant frequency by simply changing the patch length. However, antennas of this type have a relatively narrow bandwidth, which can result in performance/efficiency degradation. Nevertheless, such antennas are expected to have enough bandwidth to cover at least a single $8 \mathrm{MHz}$ channel with a suitable resonant frequency $(754 \mathrm{MHz}$, single-frequency network in Portugal).

\section{Bandwidth of Double-Dielectric Patch Antenna}

As discussed in Section 2, in order to fulfill the bandwidth requirements, we chose air as the second dielectric in the double-dielectric substrate of the patch antenna. We aimed at obtaining antenna bandwidth of at least $8 \mathrm{MHz}$ operating at the center frequency of $754 \mathrm{MHz}$.

The bandwidth of a patch antenna above a dielectric substrate depends on the dimensions of the patch and substrate permittivity. A number of approximate formulas for antenna bandwidth are available from the literature. Here, we based our bandwidth estimation on the approach developed in [10].

Considering a resonant half-wavelength patch antenna with moderate patch width $W_{p}$ satisfying $0.35<W_{p} / \lambda_{0}<2$, where $\lambda_{0}$ is the free space wavelength, the relative antenna bandwidth can be estimated [10] as

$$
B W \approx \frac{1}{Q_{\text {rad }}} \approx \frac{4 h}{W_{p} \sqrt{\varepsilon_{r, e q}}\left(\frac{W_{p}}{\lambda_{0}}-\frac{2}{\pi^{2}}\right)},
$$

where $Q_{\text {rad }}$ is the radiation quality factor of the antenna, $\varepsilon_{r, e q}$ is the equivalent relative substrate permittivity, and $h$ is the substrate thickness. From this formula, increasing $h$ and decreasing $\varepsilon_{r, e q}$ resulted in a larger bandwidth for an antenna with a given patch width.

As the air has a lower dielectric constant $\left(\varepsilon_{r}=1\right)$ compared to the average dielectric constant of the patch substrate (FR4), combining an air layer with a dielectric layer resulted in a reduction in the effective dielectric constant of the double-dielectric substrate (FR4 + air). Such a compound substrate is characterized by an equivalent relative permittivity $\varepsilon_{r, e q}$ that can be estimated by the following formula:

$$
\varepsilon_{r, e q}=\frac{1+r}{1+\frac{r}{\varepsilon_{r}}}
$$

where $\varepsilon_{r}$ is the relative permittivity of the FR4 substrate, and $r$ is a parameter determined by the thicknesses of the substrate and air layers $h_{s}$ and $h_{a}$. In plate-capacitor approximation, $r \approx \frac{h_{s}}{h_{a}}$. A more accurate result could be obtained with the conformal mapping approach [10], in which

$$
r=\frac{d_{s}}{d_{a}}
$$

where, $d_{s}=\frac{K\left(k_{s}\right)}{K^{\prime}\left(k_{s}\right)}, d_{a}=\frac{K\left(k_{a}\right)}{K^{\prime}\left(k_{a}\right)}-d_{s}$, with $k_{s}=1 / \cosh \frac{\pi W_{p}}{4 h_{s}}, k_{a}=1 / \cosh \frac{\pi W_{p}}{4\left(h_{s}+h_{a}\right)}$, and $K(x)$ is the complete elliptic integral of the first kind [11]. On the one side, when $h_{s} \ll h_{a}, r \rightarrow 0$ and the equivalent permittivity approaches the permittivity of air. On the other side, when $h_{s} \gg h_{a}, r \rightarrow+\infty$ and the equivalent permittivity approaches the permittivity of the substrate. In our case, as shown in Section 4 , air height $h_{a}$ reached $3.2 \mathrm{~mm}$.

\section{Configuration of Proposed Double-Dielectric Antenna}

The geometry of the proposed microstrip antenna is shown in Figure 1. It was expected to achieve $3 \mathrm{~dB}$ more gain by narrowing the beamwidth in the elevation direction as long as we doubled the number of patches. The antenna, consisting of three patches placed above a double-layer dielectric 
substrate, resulted in a good trade-off between size and gain. The three units increased the antenna gain by about $4.7 \mathrm{~dB}$. It was fed by a microstrip transmission line through a $50 \Omega$ SMA connector. Series-fed circuits were used to drive the three consecutive patches. The distance between the centers of the patches in the array equaled $199.0 \mathrm{~mm}\left(\sim \lambda_{0} / 2\right.$ at the operation frequency). All patches were driven in phase because the radiation amplitude and phase at each patch were determined by the cumulative transmission characteristics of the preceding microstrip lines and patches. The connecting lines had the characteristic impedance of $100 \Omega$ and no influence on the value of the central frequency [12].

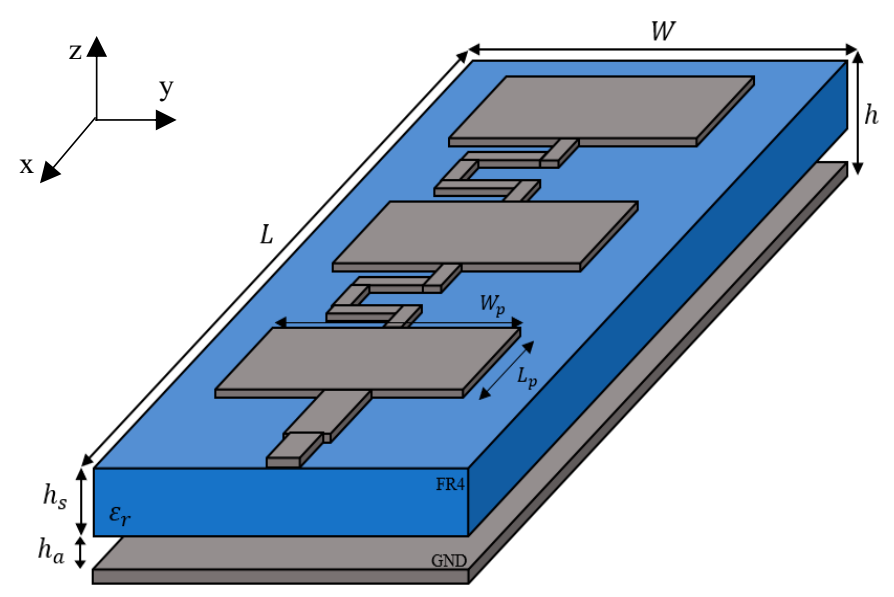

Figure 1. Geometry of double-dielectric microstrip antenna.

We followed a three-step approach. First, we theoretically designed the proposed antenna; second, we simulated the antenna behavior with the CST Microwave Studio electromagnetic simulator; third, we constructed and validated the antenna with some measurements.

On the basis of (1) and (2), we determined $h_{s}$ and $h_{a}$. The upper dielectric was composed of FR4 with thickness $h_{s}=1.6 \mathrm{~mm}$, relative permittivity $\varepsilon_{r}=4.1$, and loss tangent $\tan \delta=0.015 @ 1 \mathrm{GHz}$. The lower dielectric was composed of air with thickness $h_{a}=3.2 \mathrm{~mm}$ and relative permittivity equal to unity. Section 3 outlines how the height of the antenna influenced bandwidth.

From the desired central frequency $f_{c}=754 \mathrm{MHz}$, we estimated each patch dimension $W_{p}$ and $L_{P}$ using the following equations [13]:

$$
\begin{gathered}
W_{p}=\frac{\sqrt{2}}{2 f_{\mathcal{c}} \sqrt{\varepsilon_{0} \mu_{0}} \sqrt{\varepsilon_{r, e q}+1}} \\
L_{P}=\frac{1}{2 f_{c} \sqrt{\varepsilon_{0} \mu_{0}} \sqrt{\varepsilon_{r e f f}}}-2 \Delta L,
\end{gathered}
$$

where $\Delta L$ is given by

$$
\Delta L=\frac{0.412 h\left(\varepsilon_{\text {reff }}+0.3\right)\left(\frac{W_{p}}{h}+0.264\right)}{\left(\varepsilon_{r e f f}-0.258\right)\left(\frac{W_{p}}{h}+0.8\right)},
$$

and $h$ is the total height of the antenna, equal to $h_{s}+h_{a}$. All geometric parameters are listed in Table 1 .

Table 1. Geometric parameters of proposed double-dielectric microstrip antenna.

\begin{tabular}{cccccccc}
\hline $\begin{array}{c}\text { Frequency } \\
(\mathbf{M H z})\end{array}$ & $\varepsilon_{r, e q}$ & $\begin{array}{c}W \\
(\mathbf{m m})\end{array}$ & $\begin{array}{c}\boldsymbol{L} \\
(\mathbf{m m})\end{array}$ & $\begin{array}{c}W_{p} \\
(\mathbf{m m})\end{array}$ & $\begin{array}{c}\mathbf{L}_{p} \\
(\mathbf{m m})\end{array}$ & $\begin{array}{c}\boldsymbol{L}_{s} \\
(\mathbf{m m})\end{array}$ & $\begin{array}{c}h_{a} \\
(\mathbf{m m})\end{array}$ \\
\hline 754 & 1.7 & 270 & 673 & 180 & 161.68 & 1.6 & 3.2 \\
\hline
\end{tabular}


Each of these three patches had a size of $W_{p} \times L_{p}=180 \times 161.7 \mathrm{~mm}^{2}$. The overall size of the antenna was $W \times L=27.0 \times 67.3 \mathrm{~cm}^{2}$.

Figure 2 shows simulated reflection coefficient $S_{11}$ of the antenna. Assuming a return loss (RL) greater than $10 \mathrm{~dB}$ as the well-accepted criterion of good impedance matching, the proposed antenna was well-matched at the central frequency of $754 \mathrm{MHz}$ and in channel 56 [750-758] MHz. The simulated bandwidth was $17 \mathrm{MHz}$, obtained at $10 \mathrm{~dB}$ return loss.

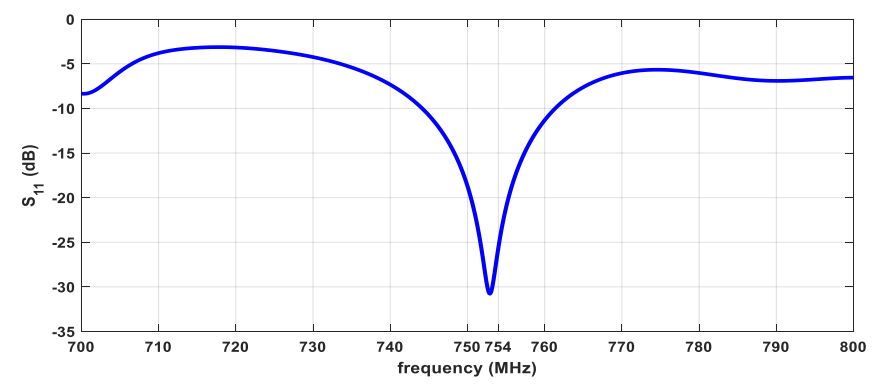

Figure 2. Simulated reflection coefficient $\left(\mathrm{S}_{11}\right)$ of proposed array antenna.

The line length connecting patches had high impedance, and its length was equal to $164.0 \mathrm{~mm}$. In total, the electrical length of the line between each consecutive patch was equal to the wavelength divided by $2, \lambda / 2$. Therefore, the input impedance of the linear array is equivalent to the parallel connection of three patch antennas. A $\lambda / 4$ transformer was then used to convert antenna input impedance into $50 \Omega$. The $\lambda / 4$ transformer line had a length of $73 \mathrm{~mm}$ and a width of $24 \mathrm{~mm}$. The $50 \Omega$ microstrip line attached to the SMA connector had a width of $17.5 \mathrm{~mm}$.

A gain value of $11.3 \mathrm{dBi}$ was obtained in the direction of the maximal radiation, which was a reasonable value since the linear array was composed of the three single patches. The gain in function of frequency is shown in Figure 3. The proposed antenna gain decreased above channel 56, as Figure 3 shows. Array efficiency was also simulated, achieving $92 \%$ at the central frequency, as shown in Figure 4 . The efficiency peak occurred at $754 \mathrm{MHz}$ and decreased at adjacent channels.

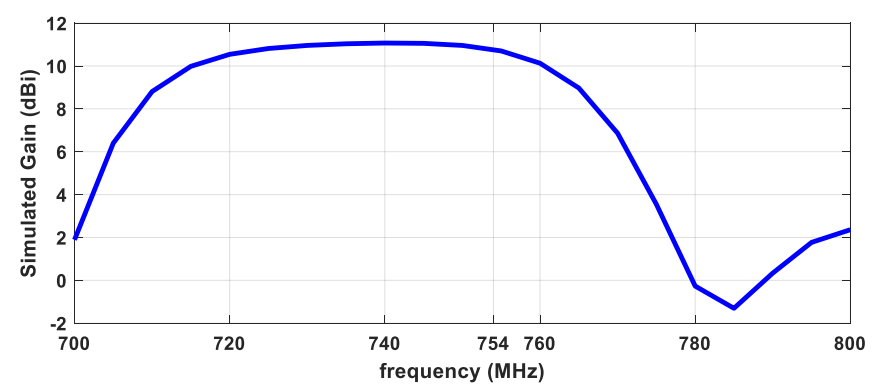

Figure 3. Simulated antenna gain over frequency.

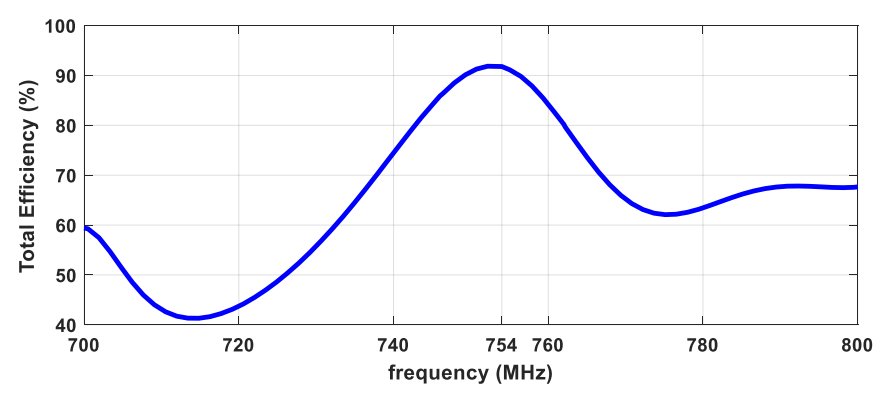

Figure 4. Simulated antenna efficiency. 


\section{Experimental Measurements}

The last step consisted of constructing the proposed antenna in order to validate our design. Thus, we checked all obtained simulation results and found the real antenna performance. The proposed double-dielectric linear array of three patch elements is illustrated in Figure 5. The bottom of Figure 5 shows the input SMA connector. This female connector was soldered to a $50 \Omega$ microstrip line. The other parts that are also visible in the figure are the $\lambda / 4$ transformer, the three radiating patches, and the connecting lines between patches.

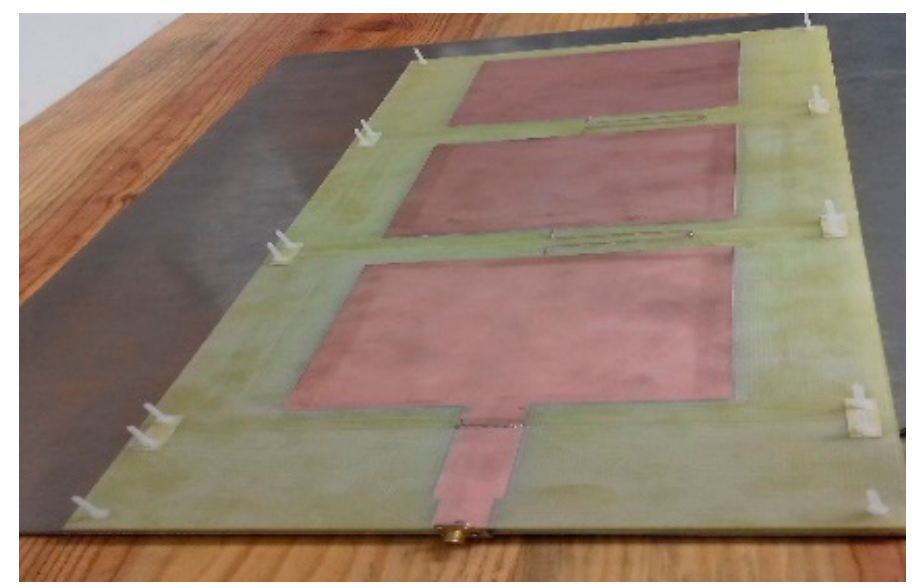

Figure 5. Construction of double-dielectric linear array.

The base of substrate FR4 was raised with plastic M3 screws at a distance of $3.2 \mathrm{~mm}$ from the ground plane made of aluminum foil with $2.0 \mathrm{~mm}$ thickness. Experimental results of reflection coefficient $S_{11}$ were obtained by measuring with a vector network analyzer (VNA), as shown in Figure 6. The center antenna frequency was clearly very close to $754 \mathrm{MHz}$, and at this frequency, $\mathrm{S}_{11}$ was less than $-20 \mathrm{~dB}$. We observed good agreement between the simulations and the measurements. However, from the practical measurements, we obtained a slightly greater bandwidth of about $25 \mathrm{MHz}$. The simulated and experimental radiation patterns obtained, in the anechoic chamber, of our proposed antenna are presented in Figure 7. The experimental and simulation diagrams were very similar. Nevertheless, there was a small difference of $7^{\circ}$ in the radiation diagrams in the vertical plane. This very small rotation in the radiation diagram was caused by a misalignment between the transmitting and receiving antennas.

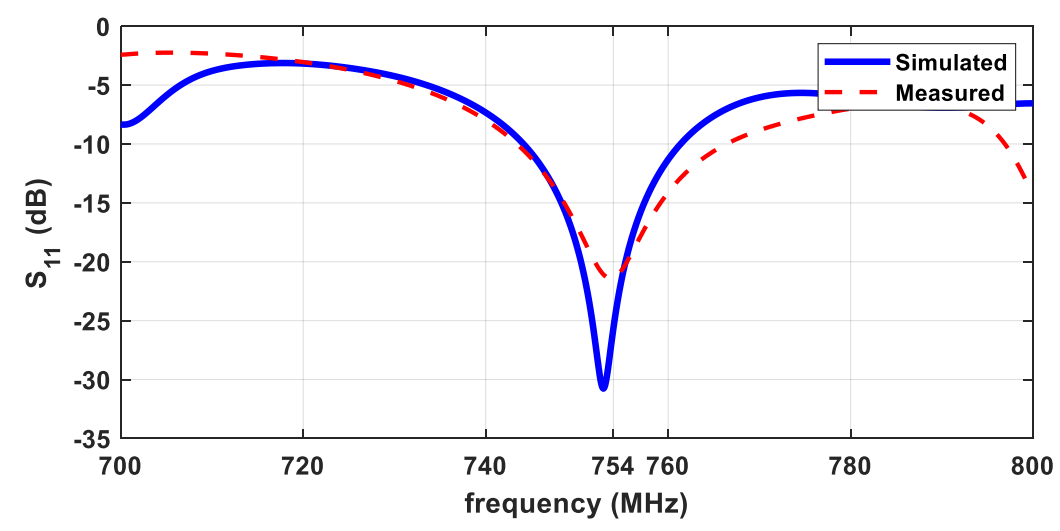

Figure 6. Measured and simulated $S_{11}$ of proposed double-dielectric antenna. 


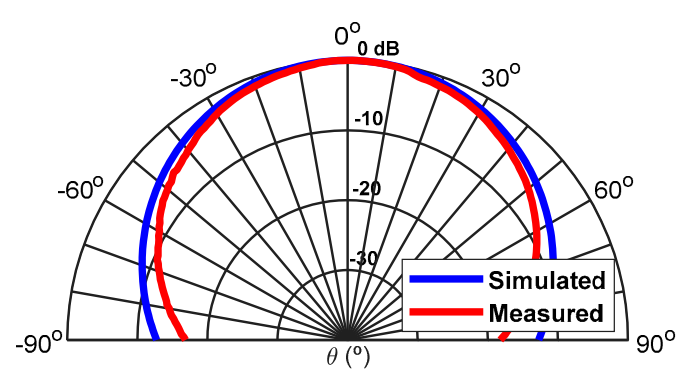

(a)

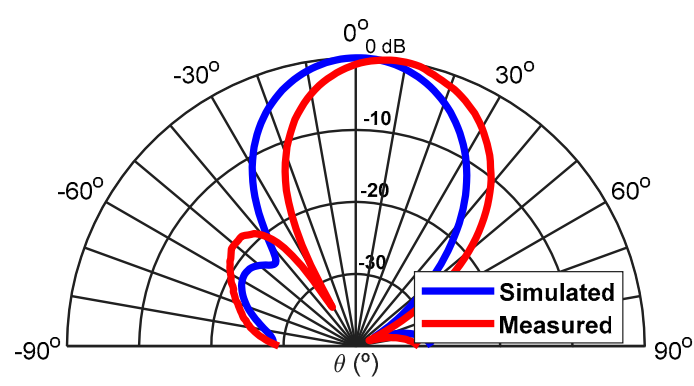

(b)

Figure 7. Simulated and measured radiation patterns. (a) $\phi=90^{\circ} ;$ (b) $\phi=0^{\circ}$.

The simulated and measured values of the gain were very similar, 11.3 and $10.5 \mathrm{dBi}$, respectively. The vertical/elevation plane $\left(\phi=0^{\circ}\right)$ had half-power bandwidth (HPBW) of $30^{\circ}$ and the horizontal/azimuth plane $\left(\phi=90^{\circ}\right)$ had a HPBW of $65^{\circ}$. These results proved that the proposed antenna had fan-beam radiation characteristics.

We also measured antenna input impedance through its input SMA connector, which was equal to $Z_{\mathrm{A}}=55.9-\mathrm{j} 10.4 \Omega$ at the central frequency, as shown in the Smith chart in Figure 8. The Smith chart was drawn from 700 to $800 \mathrm{MHz}$. There was clear mismatching at frequencies away from the desired central frequency in the simulation and experimental curves.

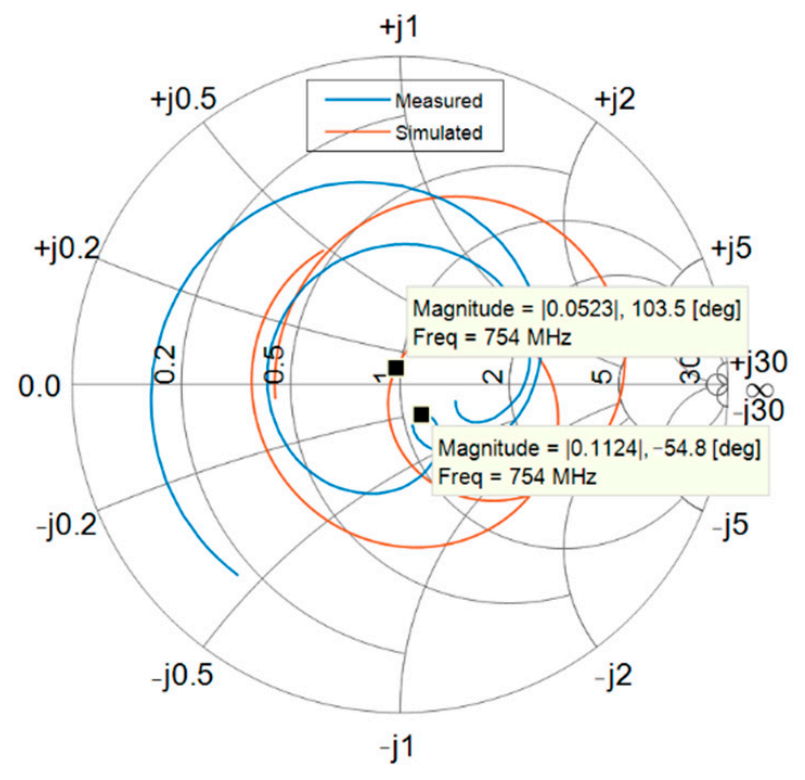

Figure 8. $\mathrm{S}_{11}$ measurements in Smith chart.

\section{Conclusions and Future Work}

This paper presented and discussed a novel practical digital TV antenna that is suitable for indoor environments. The antenna can be placed on a wall, for example, since it is very thin-about $6.8 \mathrm{~mm}$ including the aluminum foil. The upper dielectric is composed of FR4 with thickness $h_{s}=1.6 \mathrm{~mm}$, relative permittivity $\varepsilon_{r}=4.1$, and $\tan \delta=0.015 @ 1 \mathrm{GHz}$. The lower dielectric is composed of air, with thickness $h_{a}=3.2 \mathrm{~mm}$ and relative permittivity equal to unity. The designed antenna has gain of $10.5 \mathrm{dBi}$, efficiency of $92 \%$, and bandwidth of $25 \mathrm{MHz}$ at the $10 \mathrm{~dB}$ return loss level. The proposed antenna overcomes the narrowband characteristics of common microstrip antennas, is mechanically robust, and can be connected to a coaxial cable without requiring any extra electronic components or devices such as baluns. 
The proposed antenna has huge potential to be replicated and rearranged in an array. By adding electronic control to such an array, it is possible to perform smart beamforming in the azimuth plane, enabling new functionalities for DTV. An array of such antennas is quite useful for reducing self-interference caused by secondary transmitters in a single-frequency network (SFN) configuration. The antenna can also be spatially replicated to cover other applications in 5G broadcasting environments [14]. Another advantage of our proposed antenna is its bandwidth of $25 \mathrm{MHz}$, avoiding interference in the frequency domain. Although the antenna dimensions may seem large ( 67.3 by $27.0 \mathrm{~cm}$ ), the experimental demonstration presented here proved the main concept. Further reduction in the dimensions can be achieved by using reflectors or metamaterials with magnetic response, which is reserved for future work. This antenna can be used to receive any modern digital TV signals such as those specified in DVB-T/T2, ISDB-T, or ATSC standards.

Author Contributions: Antenna research and design, A.N., P.M., T.V., and J.M.; simulation, P.M. and T.V.; theoretical formulation, J.M. and S.M.; laboratory and field tests, A.N. and P.M.; methodology, A.N. and J.M.; writing, A.N., T.V., J.M., and S.M.; writing-review and editing, A.N. and J.M.; coordination, A.N. All authors have read and agreed to the published version of the manuscript.

Acknowledgments: This work is funded by FCT/MCTES through national funds and when applicable cofunded EU funds under the project UIDB/50008/2020-UIDP/50008/2020.

Conflicts of Interest: The authors declare no conflict of interest.

\section{References}

1. Yeh, J.-T.; Liao, W.-J.; Chang, S.-H. Compact internal antenna for handheld devices with comprehensive DTV band coverage. IEEE Trans. Antennas Propag. 2014, 62, 3998-4007. [CrossRef]

2. Polak, L.; Kaller, O.; Klozar, L.; Prokopec, J. Influence of mobile network interfering products on DVB-T/H broadcasting services. In Proceedings of the IFIP Wireless Days (WD), Dublin, Ireland, 21-23 November 2012.

3. Van Trinh, T.; Kim, G.; Kim, J.; Jung, C.W. Wideband internal PIFA-loop antenna designed on the bezel of digital television applications for UHF band. IET Electron. Lett. 2018, 54, 1260-1262. [CrossRef]

4. Gamec, J.; Repko, M.; Gamcová, M.; Gladišová, I.; Kurdel, P.; Nekrasov, A.; Fidge, C. Low profile sinuous Slot antenna for UWB sensor networks. Electronics 2018, 8, 127. [CrossRef]

5. Huang, C.Y.; Jeng, B.M.; Yang, C.F. Wideband monopole antenna for DVB-T applications. IET Electron. Lett. 2008, 44, 1448-1450. [CrossRef]

6. Luadang, B.; Phongcharoenpanich, C. Unidirectional bowtie array antenna with incision gap for digital video broadcasting-T2 base station. IET Microw. Antennas 2015, 9, 1087-1095. [CrossRef]

7. Ballado, A.H.; Cruz, J.D.; Sejera, M.P.; Ingco, W.M.; Juan, C.M.; Nanao, D.N.; Navarro, J.A. Design of indoor microstrip TV antenna for integrated services digital broadcast-Terrestrial (ISDB-T) signal reception at $677 \mathrm{MHz}$. In Proceedings of the International Conference on Humanoid, Nanotechnology, Information Technology, Communication and Control, Environment and Management (HNICEM), Puerto Princesa Palawan, Philippines, 12-16 November 2014.

8. Pozar, D.M.; Kaufman, B. Increasing the bandwidth of a microstrip antenna by proximity coupling. IET Electron. Lett. 1987, 23, 368-369. [CrossRef]

9. Lau, N.U.; Sloan, R. Broadband millimeter-wave suspended microstrip antenna array. In Proceedings of the 2003 IEEE Antenna and Propagation Society International Symposium, Columbus, OH, USA, 22-27 June 2003.

10. Ikonen, P.M.; Maslovski, S.I.; Simovski, C.R.; Tretyakov, S.A. On artificial magneto-dielectric loading for improving the impedance bandwidth properties of microstrip antennas. IEEE Trans. Antennas Propag. 2006, 54, 1654-1662. [CrossRef]

11. Yoon, Y.J.; Kim, B. A New Formula for Effective Dielectric Constant in Multi-Dielectric Layer Microstrip Structure. In Proceedings of the IEEE 9th Topical Meeting on Electrical Performance of Electronic Packaging (Cat. No.00TH8524), Scottsdale, AZ, USA, 6 August 2000.

12. Yuan, T.; Yuan, N.; Li, L.W. A novel series-fed taper antenna array design. IEEE Antennas Wirel. Propag. Lett. 2008, 58, 362-365. [CrossRef] 
13. Balanis, C.A. Antenna Theory, Analysis and Design: From Theory to Practice, 4th ed.; Wiley: Hoboken, NJ, USA, 2016.

14. Miao, W.; Luo, C.; Zhao, Z. Lightweight 3-D Beamforming Design in 5G UAV Broadcasting Communications. IEEE Trans. Broadcast. 2020, 66, 515-524. [CrossRef]

Publisher's Note: MDPI stays neutral with regard to jurisdictional claims in published maps and institutional affiliations.

(C) 2020 by the authors. Licensee MDPI, Basel, Switzerland. This article is an open access article distributed under the terms and conditions of the Creative Commons Attribution (CC BY) license (http://creativecommons.org/licenses/by/4.0/). 